\title{
¿A QUÉ PARADIGMA DE GESTIÓN DE LA NATURALEZA ADSCRIBE LA NORMATIVA PERUANA SOBRE CALIDAD AMBIENTAL?
}

\section{PERUVIAN LAWS ON ENVIRONMENTAL QUALITY: WHICH PARADIGM EXPLAINS THEM?}

\author{
Edgar Sánchez Infantas ${ }^{1}$ y Mariza Falero Sánchez ${ }^{2}$
}

\begin{abstract}
Resumen
Se ha llevado a cabo un análisis del contenido de la legislación peruana sobre calidad ambiental mediante la evaluación de los conceptos que usa en tres niveles: la definición de los objetivos generales de la gestión, la definición de la naturaleza del ecosistema a gestionar y el establecimiento de objetivos específicos e instrumentos para la gestión. Se ha evidenciado una contradicción entre una concepción del ecosistema que está muy cerca de lo que es un sistema complejo adaptativo y los instrumentos que se usan para su gestión que no reconocen esa condición. La contradicción puede ser consecuencia de una escasa reflexión epistemológica en la elaboración de la normativa ambiental estudiada aunque también es posible que sea manifestación de un proceso de competencia entre el paradigma que considera la naturaleza como si estuviera en estado estacionario y aquel que la considera como una entidad en evolución.

Palabras clave: Legislación ambiental, Calidad ambiental, Paradigma, Diversidad, Gestión, Participación.
\end{abstract}

\begin{abstract}
Peruvian laws on environmental quality have been analyzed by means of content analysis. Three levels have been involved: general objectives of ecosystem management, the nature of the ecosystem to be managed and the specific objectives and instruments used by the ecosystem management. Peruvian laws conceptualize the ecosystem almost as a complex adaptive system but the specific management objectives and the instruments used to manage the ecosystem do not take into consideration such approach; instead, management instruments seem to assume that the ecosystem is in steady state. These inconsistencies may be a consequence of nonsufficient epistemological reflection when the laws were elaborated or may be the consequence of a competitive process between the paradigm that sees nature as being in a steady state and the paradigm that sees nature as evolving permanently.

Key words: Environmental laws, Environmental quality, Paradigm, Diversity, Management, Involvement.
\end{abstract}

\section{Introducción.}

De acuerdo a lo que plantea Lakatos (1989) el desarrollo de una disciplina científica supone la existencia de Programas de Investigación alternativos que intentan explicar un conjunto de datos desde perspectivas diferentes. Una idea parecida es la que antes planteó Kuhn (2006) mediante el concepto de Paradigma, el mismo que ordena el trabajo científico en la medida en que establece no sólo métodos y procedimientos sino que incluso define qué es pertinente estudiar.

Existe en ambos conceptos una referencia a un núcleo central de pensamiento que ordena todo el quehacer y que no se somete a contrastación con hechos puntuales - esto último es lo que Kuhn denomina heurística negativa -. Si bien es cierto hay diferencias entre los conceptos de paradigma y de programa de investigación científica - en particular con referencia al modo en que se cambia de paradigma o de programa - es evidente que esta especie de cosmovisión que aparece en ambos conceptos es el eje ordenador del paradigma o del programa.

Ahora bien, tanto Kuhn como Lakatos tenían como preocupación fundamental el desarrollo de disciplinas científicas básicas, en particular la física. En el presente caso se intenta usar esta aproximación para evaluar un área más bien aplicada del saber ecológico; aquella que tiene que ver con la normativa legal vigente en el Perú de ahora relacionada a la calidad ambiental.

La preocupación no es sólo de carácter académico; en realidad interesa conocer qué tan bien resulta la 
gestión ambiental que se está llevando a cabo usando la plataforma conceptual que actualmente se usa. Evidentemente una cosa es conocer cuál es esa plataforma conceptual y otra conocer la efectividad de la misma en la gestión ambiental cotidiana. Es el primero de estos aspectos el que se aborda en el presente caso, es decir: ¿cuál es el paradigma al cual adscribe la legislación peruana sobre calidad ambiental?

La pertinencia de juzgar a la normativa ambiental desde la perspectiva del paradigma conceptual al que adscribe se basa en el señalamiento que hiciera Weber (1951, citado por March, 2005) y que permite diferenciar varios niveles de racionalidad. En el presente caso interesa señalar que la normativa ambiental - al igual que muchas técnicas y procedimientos - se encuentra dentro de lo que Weber denomina racionalidad instrumental. Ésta, sin embargo, sólo tiene sentido como forma de hacer efectiva una determinada cosmovisión que Weber denomina racionalidad teórica. Por tanto, resulta de interés conocer si existe coherencia entre la racionalidad teórica a la que adscribe la legislación ambiental peruana sobre calidad y la racionalidad instrumental que implementa.

La indagación que se desarrolla en el presente caso cobra interés además pues, como han mostrado Holling (1998) y Chapin et al. (2009), asistimos en el presente momento a una situación en la que existen dos paradigmas bastante bien diferenciados en la ecología: de un lado, el que entiende la naturaleza como una entidad en estado estacionario y que implementa prácticas de gestión que se inscriben en la tradición de los Máximos Rendimientos Sostenidos y, de otro lado, el que entiende a la naturaleza como una entidad en permanente evolución y en la que lo que importa es la Gestión de la Resiliencia de los ecosistemas.

\section{Materiales y métodos.}

La indagación se ha llevado a cabo tomando el modelo metodológico del Análisis de Contenido (Andreu, 1998; Berelson, 1952). Se ha identificado un conjunto de conceptos - detallados más adelante - y se ha calculado su frecuencia, entendida como el número de veces que aparece un concepto, entre las diferentes documentos objeto de análisis.

Los documentos en análisis corresponden a dispositivos legales vigentes en la normativa peruana presente y cuyo objetivo es la gestión de la naturaleza; se ha puesto especial énfasis en los dispositivos que se refieren a la calidad ambiental.

Los dispositivos legales analizados se listan en el anexo 1. Abarcan un total de 23 dispositivos legales que cubren el periodo 1991 - 2013 y se los ha escogido tomando de referencia el Compendio de la Legislación Ambiental Peruana (Perú, Ministerio del Ambiente, sf) en particular el Volumen V, Calidad
Ambiental. Es posible que la lista no sea exhaustiva, sin embargo se espera que sirva para poner de manifiesto las principales tendencias de la normativa vigente.

Los conceptos en análisis se listan en el anexo 2. El total de conceptos usados llega a 60; se los ha definido usando tres niveles de referencia, el primero de los cuales se interesa por los objetivos generales de la gestión de la naturaleza y sus recursos; el segundo nivel se refiere al modo en que se conceptualiza al ecosistema que es el objeto de manejo, es decir a la definición de su naturaleza. Finalmente, el tercer nivel se refiere a los objetivos específicos de la gestión y a los instrumentos que se usan para esa gestión. En el anexo 2, los conceptos que corresponden a los dos últimos niveles se han ordenado de acuerdo a los paradigmas a los que pertenecen.

La definición de los paradigmas y la identificación de atributos que definen cada nivel se ha hecho tomando de modelo la comparación que hace Holling (1998) de lo que denomina las “dos culturas" en ecología. Al mismo tiempo, este esquema se ha completado con el que sugieren Chapin et al., (2009) cuando comparan el manejo de la naturaleza en estado estacionario - de un lado - con la administración de la resiliencia de los ecosistemas, de otro lado.

Se han usado estas denominaciones para identificar a cada uno de los dos paradigmas en comparación: la Naturaleza Estacionaria y la Gestión de la Resiliencia.

El objeto de análisis ha sido sólo el texto resolutivo de cada norma legal, sin tomar en cuenta las consideraciones generales previas. La identificación de cada concepto en el texto ha tenido en cuenta el contexto - usualmente el párrafo - en que se encontraba el concepto en análisis.

El análisis cuantitativo de la información se ha hecho mediante una comparación simple de frecuencias de aparición de un concepto en ambos paradigmas. La evaluación se ha completado con un Análisis de Componentes Principales a partir de la matriz de Conceptos/Dispositivos legales. No se ha creído conveniente un análisis estadístico más detallado ya que lo esencial de la presente indagación no es de carácter estadístico o experimental sino más bien de índole epistemológica y conceptual.

\section{Resultados y discusión.}

Importancia relativa de los diferentes conceptos.

Una primera cosa que salta a la vista en el análisis de los conceptos que subyacen a la normativa ambiental peruana sobre calidad ambiental y gestión de la naturaleza es que de los 60 conceptos que se usaron para establecer las diferencias entre los dos paradigmas en comparación, muy pocos de ellos tienen una alta frecuencia (Figura 1). De hecho, los primeros 20 conceptos más abundantes dan cuenta de más del $90 \%$ de citas del análisis. El concepto que 


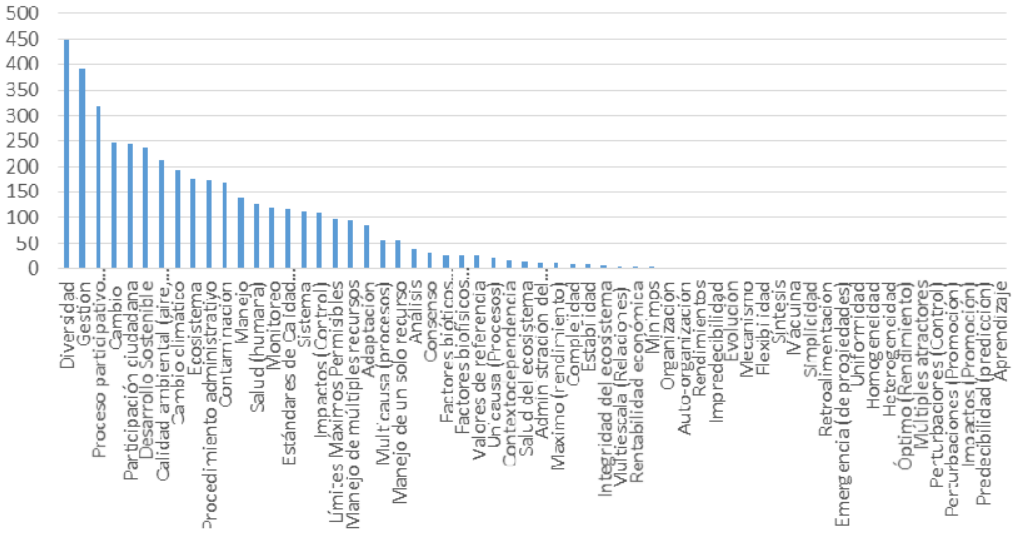

Figura 1. Frecuencia (número de citas) de aparición de los conceptos en las normas evaluadas.

aparece con más frecuencia (10.71\% de las citas totales) es Diversidad, mostrando así que es el concepto más invocado entre las normas peruanas en análisis. Le siguen los conceptos Gestión y Proceso participativo (gubernamental). En conjunto estos tres conceptos dan cuenta de un $27.68 \%$ de las citas totales. De este modo podría decirse que la normativa ambiental peruana está dirigida principalmente a la gestión de la diversidad y con una fuerte injerencia de los diferentes sectores gubernamentales.

Al mismo tiempo existe un grupo de 14 conceptos que no han sido citados ni una sola vez. Estos conceptos son: Máquina (el ecosistema entendido como una máquina), Simplicidad, Retroalimentación, Emergencia (de propiedades), Uniformidad, Homogeneidad, Heterogeneidad, Óptimo (Rendimiento), Múltiples atractores, Perturbaciones (Control), Perturbaciones (Promoción), Impactos (Promoción), Predecibilidad (predicción) y Aprendizaje. Como se ve, algunos de estos conceptos atañen a la naturaleza del ecosistema que se quiere gestionar (Emergencia, Predecibilidad, Uniformidad, a modo de ejemplo) mientras que otros se refieren a los objetivos de dicha gestión (obtener Óptimos Rendimientos, controlar las Perturbaciones, a modo de ejemplo).

Evaluación global de los conceptos agrupados por los objetivos generales de la gestión, por la naturaleza del ecosistema y por las herramientas y metas usadas en la gestión.

El total de conceptos citados se agrupó de acuerdo a tres criterios: los objetivos generales de la gestión ambiental y de los recursos, la naturaleza del ecosistema que se quiere gestionar y finalmente las herramientas que efectivamente se usan en esa gestión y las metas que se buscan. El primer criterio corresponde a una especie de declaración general de lo que la normativa ambiental peruana entiende que son los objetivos de la gestión. Sus resultados (Tabla 1,
Figura 2) muestran que es la Diversidad el concepto más citado; le sigue Gestión y luego un grupo de tres conceptos con una frecuencia muy similar: Desarrollo Sostenible, Calidad Ambiental y Cambio Climático.

Por tanto, de manera general se podría señalar que lo que la normativa ambiental peruana entiende es que la tarea de la gestión de la naturaleza es la búsqueda del desarrollo sostenible teniendo como objetos de su interés a la diversidad, la calidad ambiental y el cambio climático. Como temas complementarios aparecen la contaminación y la salud humana. Esta declaración temática aparece reiteradamente en los subsiguientes análisis. Conviene señalar que el concepto Calidad Ambiental aparece entre los objetivos generales de la gestión. Por otro lado debe decirse que siendo estos objetivos generales de la gestión no resulta pertinente separar dentro de qué paradigma se encuentra cada uno de ellos; esto es algo que se hace a continuación pero en referencia a la manera cómo se entiende la naturaleza del ecosistema a gestionar y las herramientas y estilos que se usan en la gestión.

Las Tablas 2 y 3 y la Figura 3 muestran que cuando se trata de la naturaleza del ecosistema a gestionar, los conceptos se distribuyen de manera que hay una marcada dominancia de conceptos asociados al paradigma de la Gestión de la Resiliencia. Esta dominancia se hace manifiesta en particular en el tipo de estructura que se supone es el ecosistema que se habrá de gestionar; así, el concepto de Sistema supera largamente al concepto de Mecanismo (289 y 1 citas, respectivamente). Esto implica que la normativa

Tabla 1. Frecuencia de aparición de conceptos referidos a los objetivos de la gestión ambiental y de los recursos naturales.

\begin{tabular}{lr}
\hline Concepto & No. de citas \\
\hline Diversidad & 448 \\
Gestión & 392 \\
Desarrollo Sostenible & 238 \\
Calidad ambiental & 211 \\
Cambio climático & 194 \\
Procedimientos Administrativos & 172 \\
Contaminación & 168 \\
Manejo & 138 \\
Salud humana & 126 \\
Salud del ecosistema & 13 \\
Valores de referencia & 26 \\
Rendimientos & 2 \\
\hline
\end{tabular}




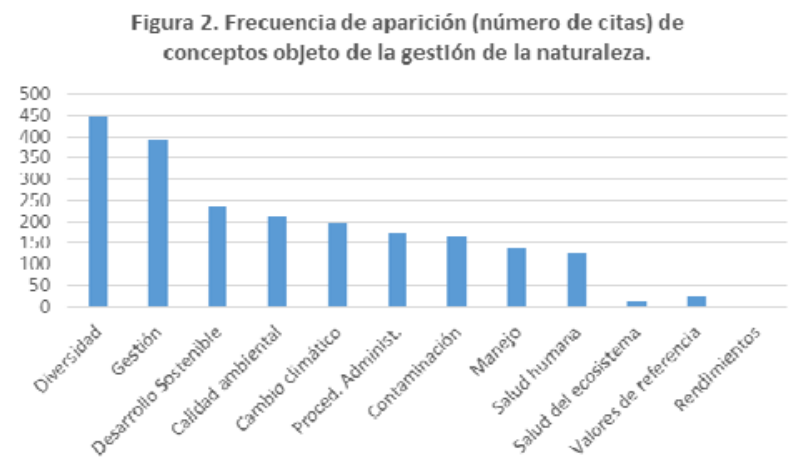

Figura 2. Frecuencia de aparición (número de citas) de conceptos objeto de la gestión de la naturaleza.

peruana reconoce correctamente que el objeto a gestionar es un sistema y no un mecanismo.

En concordancia con lo dicho, cuando el atributo que se considera es el comportamiento de este sistema en el tiempo, el concepto Cambio supera largamente al concepto Estabilidad (248 y 8 citas, respectivamente). Resulta interesante esta dominancia especialmente si se tiene en cuenta que el discurso ambientalista usualmente enfatiza en el concepto de estabilidad (De Leo \& Levin, 1997; Hull et al., 2002; Marshall, 1998) a pesar de las varias críticas que ha recibido (Odenbaugh, 2001; Green, 2006).

Adicionalmente, otros conceptos que denotan una cercanía al paradigma de la Gestión de la Resiliencia son Complejidad (14 citas) en oposición a

Tabla 2. Frecuencia de aparición de conceptos referidos a la naturaleza del ecosistema a gestionar; se comparan los conceptos - y sus frecuencias de aparición - dentro de los dos paradigmas en evaluación: la Naturaleza Estacionaria y la Gestión de la Resiliencia.

\begin{tabular}{|c|c|c|c|c|}
\hline \multirow{2}{*}{$\begin{array}{l}\text { Atributos } \\
\text { Tipo de estructura }\end{array}$} & \multicolumn{2}{|c|}{ Naturaleza Estacionaria } & \multicolumn{2}{|l|}{ Gestión de la Resiliencia } \\
\hline & Máquina & 0 & Ecosistema & 176 \\
\hline & Mecanismo & 1 & Sistema & 113 \\
\hline Tipo de causalidad & Unicausa (Procesos) & 21 & Multicausa (procesos) & 54 \\
\hline Variables & Factores bióticos & & biofísicos & \\
\hline importantes & (Organización por) & 28 & (Organización por) & 26 \\
\hline Nivel de & & & & \\
\hline complejidad & Simplicidad & 0 & $\begin{array}{l}\text { Complejidad } \\
\text { Multiescala (Relaciones) }\end{array}$ & $\begin{array}{l}9 \\
5\end{array}$ \\
\hline $\begin{array}{l}\text { Procesos que } \\
\text { operan }\end{array}$ & Organización & 2 & $\begin{array}{l}\text { Auto-organización } \\
\text { Emergencia } \\
\text { propiedades) }\end{array}$ & 2 \\
\hline & & & Retroalimentación & 0 \\
\hline $\begin{array}{l}\text { Comportamiento } \\
\text { en el tiempo }\end{array}$ & Estabilidad & 8 & Cambio & 246 \\
\hline & & & Evolución & 2 \\
\hline & & & Múltiples atractores & 0 \\
\hline & & & Aprendizaje & 0 \\
\hline Predecibilidad & $\begin{array}{l}\text { Predecibilidad } \\
\text { (predicción) }\end{array}$ & 0 & Impredecibilidad & 2 \\
\hline Diferenciación & Homogeneidad & 0 & Heterogeneidad & 0 \\
\hline & Uniformidad & 0 & & \\
\hline
\end{tabular}

Simplicidad (0 citas) y la existencia de un tipo de causalidad referido a múltiples orígenes: Multicausalidad (54 citas) frente a Unicausalidad (21 citas). Por lo tanto, esto indica que la normativa ambiental peruana hace un reconocimiento de que el objeto de su gestión es un sistema complejo, que evoluciona en el tiempo y que las causas de esta evolución tienen múltiples fuentes. Esta descripción de lo que es el sistema a gestionar se parece bastante a lo que Boyle et al. (2001) y Holling et al. (2001) indican que es un Sistema Complejo Adaptativo. Siendo esto así, se espera que los instrumentos de gestión de los que hace uso esta normativa, se correspondan con la condición compleja y adaptativa que se le atribuye al objeto a gestionar. Esto es tema de la siguiente sección.

Cuando se analizan los objetivos específicos de la gestión y los instrumentos o herramientas que se emplean para tal gestión, se encuentra (Tablas 4 y 5 , Figura 4) una circunstancia que resulta diferente a la que se había hallado al evaluar la naturaleza del ecosistema a gestionar. Un vistazo general a la Figura 4 muestra que los atributos están distribuidos de modo más o menos parejo entre los dos paradigmas que se están comparando, cosa que contrasta con la marcada dominancia del paradigma de la Gestión de la Resiliencia cuando lo que se evalúa es la naturaleza del ecosistema a gestionar (Figura 3).

Un análisis más detallado muestra que la paridad entre ambos paradigmas se da en el tipo de participación que propician: Participación gubernamental -los diferentes sectores del gobierno- (318 citas) frente a la Participación ciudadana (275 citas). Siendo así que este tipo de participación es uno de los aspectos críticos de la diferencia entre ambos paradigmas como lo muestran Chapin et al. (2009), este casi empate es significativo. De hecho en el paradigma de la Naturaleza Estacionaria, son los técnicos y los funcionarios los que establecen los objetivos de la gestión de la naturaleza, sus ecosistemas y sus recursos; en el 0 paradigma de la Gestión de la

0 Resiliencia, en cambio, es un consenso intersubjetivo entre técnicos y/o funcionarios y los actores sociales involucrados lo que fija los objetivos de la gestión (Walker et al., 2002).

Un segundo aspecto en el que existe una cierta paridad se refiere a los instrumentos que se 


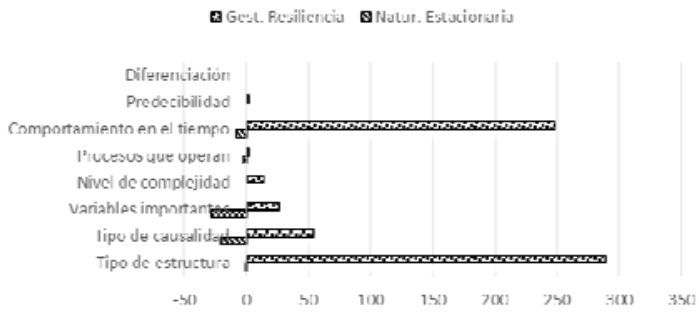

Figura 3. Frecuencia de conceptos asociados a cada paradigma: Naturaleza del ecosistema.

usan en la gestión; existen 214 citas del grupo de conceptos Estándares de Calidad Ambiental (ECAs) y Límites Máximos Permisibles (LMPs) frente a 220 citas para el grupo que incluye a los conceptos Monitoreo, Adaptación, Flexibilidad y Contextodependencia.

Detrás de los conceptos de ECAs y LMPs está la idea de que es posible encontrar valores de referencia que puedan tener valor para un gran número de casos, es decir, se puedan generalizar. Naturalmente esto es válido cuando se asume que la naturaleza se comporta de tal manera que tiende hacia un estado estacionario, el cual, una vez alcanzado, se mantendrá indefinidamente. Este único atractor, además, se supone es más bien universal - al menos para un tipo particular de ecosistemas - y se puede usar entonces como el estado saludable del ecosistema. Como indican De Leo \& Levin (1997) esta es la idea de que hay un sólo "estado saludable" del ecosistema y que todos los demás tienen carácter patológico, idea que además es consecuencia del punto de vista que impuso Clements sobre la evolución sucesional de un ecosistema y que como ha indicado Marshall (2002) tiene más de contenido metafísico que de constatación empírica.

La visión alternativa parte del reconocimiento de que el ecosistema evoluciona, es dinámico, es un sistema abierto y tiene comportamientos emergentes y sorpresivos (Kay et al, 1999) y que por tanto, en su gestión se debe involucrar a los conceptos de Monitoreo, Adaptación, Flexibilidad y Contextodependencia.

Se presenta entonces una incongruencia ya que si como se ha visto en la Figura 3 - la legislación peruana reconoce que la naturaleza del ecosistema a gestionar es tal que es un sistema complejo, que evoluciona en el tiempo y que las causas de esta evolución tienen múltiples fuentes, sólo tiene sentido que los instrumentos que se emplean en la gestión sean los referidos a la
Tabla 3. Frecuencias de conceptos referidos a la naturaleza de los ecosistemas dentro de cada paradigma en análisis. Se han agrupado los conceptos por cada atributo usando la información de la Tabla 2.

\begin{tabular}{llrlr}
\hline Atributo & Naturaleza Estacionaria & Gestión de la Resiliencia \\
\hline Tipo de estructura & Mecanismo & 1 & Sistema & 289 \\
Tipo de causalidad & Unicausalidad & 21 & Multicausalidad & 54 \\
Variables importantes & V. biológicas & 28 & V. biofísicas & 26 \\
Nivel de complejidad & Simplicidad & 0 & Complejidad & 14 \\
Procesos que operan & Organización & 2 & Auto-organización & 2 \\
Comportamiento en el tiempo & Estabilidad & 8 & Cambio & 248 \\
Predecibilidad & Predecibilidad & 0 & Impredecibilidad & 2 \\
Diferenciación & Homogeneidad & 0 & Heterogeneidad & 0 \\
\hline
\end{tabular}


Tabla 4. Frecuencia de aparición de conceptos referidos a las herramientas y los objetivos de la gestión; se comparan los conceptos - y sus frecuencias de aparición - dentro de los dos paradigmas en evaluación: la Naturaleza Estacionaria y la Gestión de la Resiliencia.

\begin{tabular}{llrlr}
\hline Atributos & Naturaleza Estacionaria & Gestión de la Resiliencia \\
\hline Tipo de gestión & Manejo de un solo recurso & 54 Manejo de múltiples recursos & 94 \\
& & Administración del ecosistema & 11 \\
Objetivo & 5 Integridad del ecosistema & 6 \\
Metas a conseguir & Rentabilidad económica & 10 Óptimo (Rendimiento) & 0 \\
& Máximos (rendimientos) & 3 & & 1 \\
Métodos & Mínimos & 39 & Síntesis \\
Instrumentos & Análisis & 117 Monitoreo & 120 \\
& Estándares de Calidad Ambiental (ECAs) & 84 \\
& Límites Máximos Permisibles (LMPs) & 97 Adaptación & 1 \\
Rol de las perturbaciones & Impactos (Control) & Clexibilidad & 15 \\
& & 111 Impactos (Promoción) & 0 \\
Tipo de participación & Perturbaciones (Control) & 0 Perturbaciones (Promoción) & 0 \\
& Proceso participativo (gobierno) & 318 Participación ciudadana & 244 \\
\hline
\end{tabular}

hecho, el referirse a la diversidad biológica alude a varias especies, cada una de las cuales puede ser un recurso; igualmente sucede con el concepto de calidad ambiental, el mismo que implica múltiples variables para su definición.

Aunque el número total de citas ha sido bastante bajo tanto para la Rentabilidad (5 citas) como para la Integridad del ecosistema (6 citas), como conceptos que definen el objetivo de la gestión de un ecosistema, el virtual empate entre ambos es también significativo de una situación contradictoria. En efecto, tanto Holling (1998) como Chapin et al. (2009) han mostrado que dentro del paradigma de la naturaleza estacionaria, el objetivo del manejo suele ser un solo recurso del cual se quiere obtener la máxima utilidad posible de modo sostenido; es el concepto de Máximo Rendimiento Sostenido, el mismo que además tiene un fuerte sesgo hacia el reconocimiento de que la fuente del valor de un recurso es el que le confiere un mercado; la rentabilidad es un concepto que captura esta idea.

De forma alternativa, el mantenimiento de la integridad del ecosistema se presenta como un objetivo más acorde con el reconocimiento de que éste es un sistema complejo que evoluciona en el tiempo y en el que resulta más útil administrar varios recursos que intentar maximizar la rentabilidad que se pueda obtener del manejo de uno sólo de éstos. Por lo tanto, el hecho de que haya un empate entre rentabilidad e integridad, como objetivos de la gestión, denota una situación contradictoria, similar a la que se presentó antes con relación al tipo de participación que la gestión propicia y a los instrumentos o herramientas de gestión, aun cuando ésta es de menor magnitud que aquellas dado el menor número de citas involucradas.

Un aspecto adicional en el que la dominancia del paradigma de la naturaleza estacionaria es evidente y que está relacionado con lo que se acaba de señalar es la oposición entre Máximos/Mínimos (13 citas) y Óptimos (ninguna cita) con relación a las metas a conseguir en la gestión de un ecosistema. La idea de obtener un Máximo Rendimiento Sostenido de la gestión de un ecosistema es sólo un caso particular de la búsqueda de maximizar o minimizar alguna función de tipo utilidad, beneficio, cosecha de biomasa o perturbación. En el caso de un sistema en el que hay múltiples atractores, en el que la emergencia y la sorpresa son siempre una posibilidad, así como la contexto-dependencia, no es posible establecer un valor único de referencia sobre el cual buscar maximizar o minimizar alguna propiedad; en este caso lo que conviene es que el sistema tenga suficiente reserva de resiliencia, pero no tanta que al final vea reducida su capacidad de evolucionar (Cumming y Collier, 2005; Folke, et al., 2010).

Un grupo de conceptos en los que la dominancia del paradigma de la naturaleza estacionaria es muy marcada tiene que ver con las actitudes frente a las perturbaciones. El Control de perturbaciones tiene un total de 11 citas frente a ninguna para la

Tabla 5. Frecuencias de conceptos referidos a las herramientas y los objetivos de la gestión de los ecosistemas dentro de cada paradigma en análisis. Se han agrupado los conceptos por cada atributo usando la información de la Tabla 4.

\begin{tabular}{llrlr}
\hline Atributo & Naturaleza Estacionaria & Gestión de la Resiliencia & \\
\hline Tipo de gestión & Manejo de un solo recurso & 54 & Administración de varios recursos & 105 \\
Objetivo & Rentabilidad & 5 Integridad del ecosistema & 6 \\
Metas a conseguir & Máximos o Mínimos & 13 Óptimos & 0 \\
Métodos & Análisis & 214 Síntesis & 1 \\
Instrumentos & ECAs y LMPs & 111 Promitoreo/Adaptación & 220 \\
Actitud ante las perturbaciones & Control & 318 Ciudadana & 0 \\
Tipo de participación & Gubernamental & & 275 \\
\hline
\end{tabular}


Promoción de perturbaciones. Chapin et al. (2009) señalan que este es otro punto en el que se diferencian marcadamente ambos paradigmas. Desde la perspectiva de la naturaleza estacionaria, al haber un solo estado estacionario hacia el cual se dirige el ecosistema, de lo que se trata en la gestión es de evitar perturbaciones que alejen al ecosistema de ese estado de referencia, es decir, la tarea es controlar las perturbaciones. Por el contrario, si no existe un solo atractor hacia el cual se dirige el ecosistema, existiendo más bien múltiples atractores (Boyle et al., 2001; Kay et al., 2001), no tiene sentido hablar de control de perturbaciones ya que el cambio entre estos atractores deja de tener carácter de perturbación para ser parte de la dinámica normal del ecosistema (Beisner et al., 2003).

Es más, el paradigma de la gestión de la resiliencia asume que lo natural de un ecosistema es que éste pase por varias fases en su evolución y que - a diferencia de lo que planteaba la idea más convencional de sucesión clementsiana - así como hay una fase de climax (fase K), existe también una fase de crisis (fase omega) en la que el ecosistema da paso a una reconstrucción de su estructura y dinámica, a la incorporación de novedades (fases alfa) y a la construcción de un nuevo ciclo adaptativo (fase r) (Cumming \& Collier, 2005). En esta visión alternativa del funcionamiento de los ecosistemas, de lo que se trata es de promover aquellas perturbaciones que garantizan su funcionamiento, es decir, aquellas que mantienen las propiedades del ecosistema, propiedades que incluyen su capacidad de evolucionar. Esta capacidad de evolución es tan básica para un ecosistema en este paradigma, que incuso se ha definido el concepto de transformabilidad para referirse a ella (Walker et al., 2004). Por tanto, al hallarse una total dominancia del control de perturbaciones sobre su promoción en la legislación peruana, está habiendo una contradicción con el hecho de haber conceptuado al ecosistema como un sistema complejo adaptativo (Tabla 3, Figura 3).

Un último tópico en el que es marcada la dominancia del paradigma de la naturaleza estacionaria es de carácter metodológico; atañe al uso del Análisis (39 citas) frente al uso de la Síntesis (1 cita) en la gestión de los ecosistemas. Las correspondientes frecuencias de citas de ambos conceptos muestran una muy marcada dominancia de un enfoque analítico, cartesiano y - por lo mismo reduccionista. En contrapartida la versión integradora - en el sentido de Holling (1998) -, que hace referencia a visiones de conjunto, está prácticamente ausente. De este modo, la visión que domina en la legislación ambiental peruana no permite ver la emergencia de características que sólo aparecen cuando todos los elementos del sistema están juntos (Ulanowicz, 2007).
De modo general, puede decirse entonces que existe una contradicción en la legislación peruana sobre calidad ambiental ya que concibe al objeto de su gestión, el ecosistema, reconociéndole propiedades de un Sistema Complejo Adaptativo y sin embargo los instrumentos que usa para implementar esta gestión si bien en alguna medida respetan esta condición, existen un grupo nada despreciable de consideraciones que se corresponden más bien con la idea de una naturaleza estacionaria.

Evaluación de los conceptos desde una perspectiva multivariada.

Los análisis anteriores se han referido a la frecuencia total de los diferentes conceptos; de este modo se analizaron las normas legales referidas a calidad como un solo todo sin hacer mayor referencia a las especificidades que pueden tener cada dispositivo legal en particular. La evaluación de estos dispositivos usando un Análisis de Componentes Principales (ACP) (Figura 5) permite identificar tanto los conceptos que ordenan el conjunto de dispositivos legales, como a la ubicación de estos dispositivos legales con relación a los conceptos.

En efecto, las correlaciones de los conceptos con el Componente Principal 1 (CP1) (Figura 6) muestran que Desarrollo Sustentable, Manejo, Procedimientos Administrativos y Diversidad son los conceptos que más relevancia tienen. Estos cuatro conceptos tienen un carácter más bien de definición de lo que se espera de una norma ligada a la gestión de la naturaleza y de sus recursos.

Las normas que mejor representan estos conceptos son (Figura 5) el DS 102-2001 (Aprobación de la Estrategia Nacional de la Diversidad Biológica),la ley 28611 (Ley General del Ambiente), el DS 086-2003 (Aprobación de la Estrategia Nacional sobre el Cambio Climático) y el DS 008-2005 (Reglamento de la Ley Marco del Sistema Nacional de Gestión Ambiental).

La asociación de estas normas con conceptos que tienen más bien un carácter general, muestra a su vez el carácter general con que han sido redactadas. En ese sentido, el primer componente principal representaría un gradiente de generalidad: en el lado positivo están las leyes de carácter general, en el lado negativo las leyes de carácter específico. Este resultado es coherente con lo que el análisis de a frecuencia total de conceptos había mostrado (Figuras 1 y 2).

Las correlaciones con el CP2 (Figura 7) muestran una separación muy clara de dos temas centrales que son preocupación de la gestión ambiental: en el lado positivo de dicho componente se tiene a los conceptos Calidad Ambiental y Salud Humana y en menor medida a Salud del Ecosistema; en el lado negativo de este componente están los conceptos Rendimientos (sostenidos), Diversidad y Manejo.

De manera general se puede decir entonces que los problemas que tradicionalmente se han asociado al 


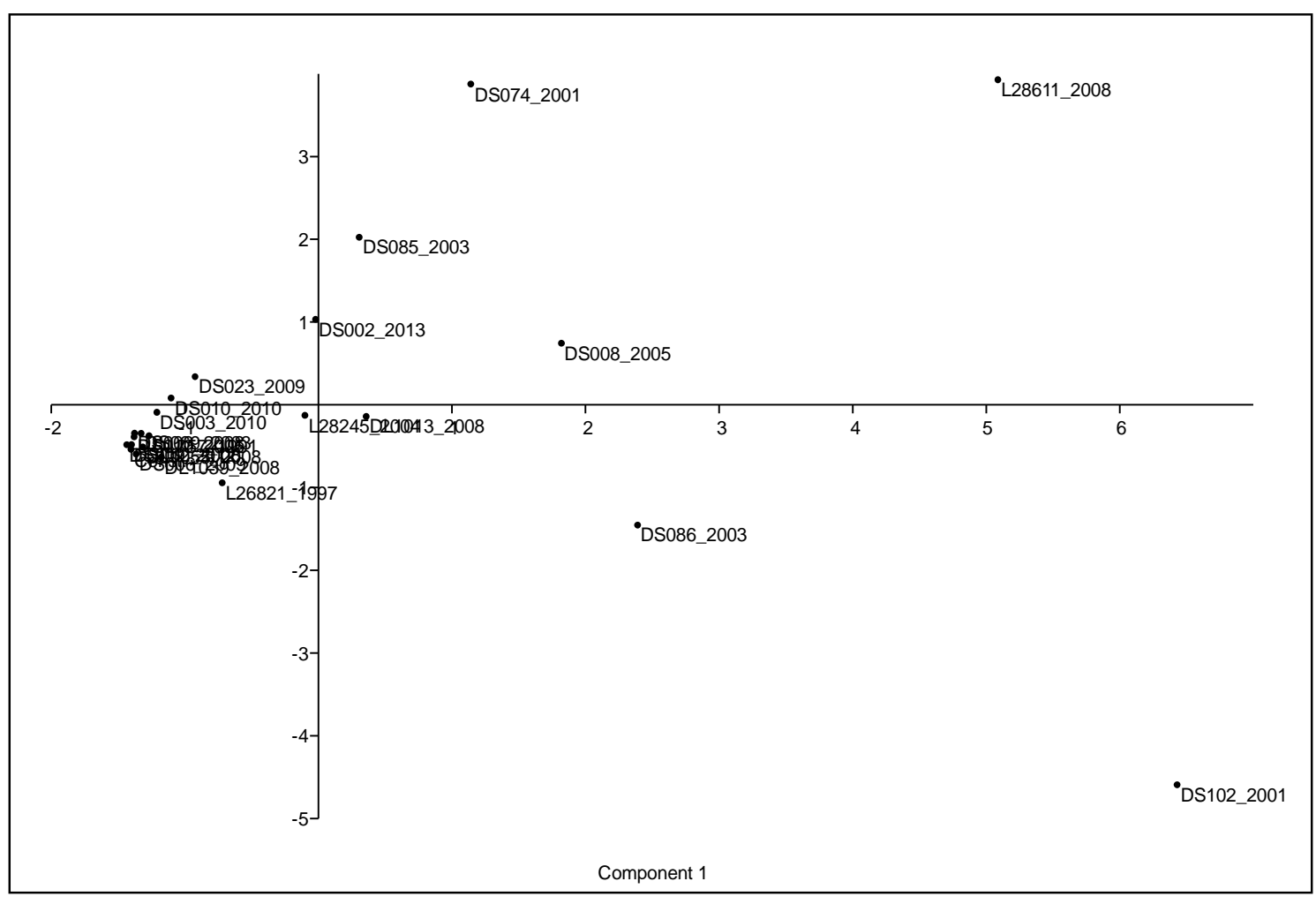

Figura 5. Análisis de Componentes Principales de los dispositivos legales peruanos sobre calidad ambiental.

denominado Frente Marrón (Calidad ambiental, Vertimientos, Emisiones, y sus efectos sobre la salud de las personas y los ecosistemas) definen el lado positivo de este componente principal. Por su parte, el lado negativo del componente está asociado a lo se ha denominado Frente Verde (Manejo de la Diversidad y los recursos naturales para obtener Rendimientos Sostenidos).

De este modo, se puede señalar que la evaluación multivariada de los dispositivos legales referidos a calidad ambiental en el Perú, muestra que estos se organizan alrededor de dos ejes centrales: el primero el más importante por explicar la mayor cantidad de la varianza - representa un gradiente de generalidad/especificidad de las normas y el segundo es un gradiente de Frente Marrón - Frente Verde, es decir una segregación temática.

Evaluación global de los resultados.

La segregación que el Análisis de Componentes Principales hace de los dispositivos legales, muestra dos ejes ordenadores que si bien ponen de manifiesto dos ejes que ordenan a los dispositivos (generalidad/especificidad $\mathrm{y}$ frente verde/frente marrón), no ayudan mucho en el análisis conceptual y su relación con los dos paradigmas en comparación.

De otro lado, si tomamos en cuenta los tres niveles de referencia que se han usado para ordenar los conceptos, puede señalarse que el primero - los objetivos generales de la gestión - es una declaración muy general de lo que la gestión quiere hacer. El hecho de que acá dominen conceptos como Diversidad, Gestión, Desarrollo Sostenible, Calidad ambiental y Cambio climático muestra un conjunto que puede servir para definir cualquier sistema de gestión ambiental y que por eso mismo no resulta posible de someter a crítica. Al mismo tiempo no parece posible señalar que a este nivel de generalidad puedan haber diferencias entre paradigmas. De este modo, se puede aceptar como válida la declaración de objetivos generales que la normativa ambiental peruana hace.

El segundo nivel de referencia tiene como objeto definir la naturaleza del ecosistema que se quiere gestionar. En la medida en que algunos de los pares de conceptos involucrados en este nivel tienen un carácter incluso ontológico (sistema frente a mecanismo, por ejemplo), resulta razonable entender que este nivel puede corresponder a lo que Weber llamaba racionalidad teórica, es decir aquella que se expresa en una cosmovisión determinada. Al mismo tiempo, el tercer nivel de referencia define objetivos específicos de la gestión e instrumentos para su obtención. Este nivel parece corresponder entonces a la racionalidad instrumental de Weber. 




Figura 6. Correlaciones de los diferentes conceptos con el componente principal 1 (CP1) del ACP de la Figura 5.

Puestas así las cosas, resulta que existe una contradicción en la legislación peruana sobre calidad ambiental ya que su racionalidad teórica considera al ecosistema como un sistema complejo adaptativo - lo que la hace afín al paradigma de la gestión de la resiliencia - en tanto que su racionalidad instrumental tiene una importante contribución de conceptos que más bien corresponden al paradigma de la naturaleza estacionaria. De hecho existe una suerte de paridad entre ambos paradigmas cuando se trata de conceptos referidos al tipo de participación (gubernamental o ciudadana), los instrumentos de gestión (ECAs y LMPs frente a Monitoreo y Adaptación) y el tipo de gestión (manejo de un recurso ó de muchos recursos). Además, con relación a las perturbaciones, es decidida la dominancia del concepto que corresponde al paradigma de la naturaleza estacionaria (control de las perturbaciones).

¿Cómo explicar esta incoherencia entre racionalidad teórica - cosmovisión - y racionalidad instrumental en la legislación peruana sobre calidad ambiental? Una posible explicación - que tiene carácter conjetural - es que no se ha dado entre los autores de la legislación analizada una discusión detallada de las implicancias de considerar al ecosistema como un sistema complejo adaptativo, discusión que mostraría, por ejemplo, la incoherencia de considerar a un ecosistema como un sistema complejo y sin embargo querer manejarlo con instrumentos como los ECAs y los LMPs. En realidad no es muy común la discusión epistemológica de los conceptos en ecología ni aún en un ámbito puramente académico, como lo señalan Nuñez y Nuñez (2005). De otro lado y de modo alternativo podría tratarse de un proceso de competencia entre ambos paradigmas, proceso en el que se ha logrado cierto acuerdo en la



Figura 7. Correlaciones de los diferentes conceptos con el componente principal 2 (CP2) del ACP de la Figura 5.

racionalidad teórica pero en el que la controversia se mantiene al nivel de la racionalidad instrumental. Incluso en esta segunda posibilidad, estaríamos también frente a una incoherencia - aunque sea parcial - entre ambas racionalidades.

Al parecer surgen al menos dos tareas pendientes a partir de lo encontrado en la presente indagación; la primera se refiere a la necesidad de desarrollar una reflexión epistemológica más detallada entre los responsables de construir la legislación ambiental, de modo de resolver las incoherencias vistas. Esta tarea alcanza no sólo a los propios funcionarios del Ministerio del Ambiente y a los legisladores sino también a las universidades que los forman.

La segunda tarea se refiere a la necesidad de escoger entre los dos paradigmas en competencia. Si prestamos atención a lo dicho por Lakatos (1989) la elección es un proceso racional que implica identificar cuál de los programas de investigación que compiten tiene exceso de contenido empírico sobre los otros programas, es decir, cuál permite ver o entender una mayor cantidad de cosas. Este puede ser un proceso más o menos largo y podría ser que las contradicciones halladas en el presente caso sean consecuencia de este proceso. Esto, sin embargo es materia de otra indagación.

\section{Conclusiones.}

- El análisis del contenido conceptual de las normas peruanas sobre calidad ambiental ha mostrado que existe un primer nivel de definición de los objetivos generales de la gestión de los ecosistemas y en el que los conceptos que aparecen con mayor frecuencia corresponden a Diversidad, Gestión, Desarrollo Sostenible, Calidad Ambiental y Cambio Climático. 


\section{¿A QUÉ PARADIGMA DE GESTIÓN DE LA NATURALEZA ADSCRIBE LA NORMATIVA PERUANA SOBRE CALIDAD AMBIENTAL?}

Julio - Diciembre 2015

- Cuando lo que se analiza es la naturaleza del ecosistema a gestionar, existe una marcada dominancia de conceptos tales como Sistema (en oposición a Mecanismo), Complejidad, Cambio y Multicausalidad de este cambio. Esto muestra que la concepción que la legislación peruana sobre calidad ambiental tiene de la naturaleza, es muy afín a lo que es un Sistema Complejo Adaptativo; en ese sentido esta cosmovisión se sitúa dentro de lo que sería el paradigma de la naturaleza evolutiva y le correspondería una gestión orientada a la administración de su resiliencia.

- Al evaluar los objetivos específicos de la gestión y las herramientas que usa, se constata una contradicción con la cosmovisión antes dicha ya que existe una casi paridad entre conceptos que pertenecen tanto al paradigma de la naturaleza estacionaria como al de la naturaleza evolutiva. En efecto la paridad se da con relación al tipo de participación que se propicia en la gestión (gubernamental o ciudadana), a los instrumentos que se usan (Estándares de Calidad y Límites Máximos permisibles en oposición al monitoreo, la adaptación y la contexto-dependencia), y el manejo de los recursos (uno sólo o conjuntos de recursos). Además existe una dominancia marcada de algunos conceptos propios del paradigma de la naturaleza estacionaria; se trata, por ejemplo del control de las perturbaciones (en oposición a su promoción), de la búsqueda de máximos o mínimos (en oposición a óptimos) y de la aplastante dominancia del análisis frente a la síntesis.

- La contradicción entre la racionalidad teórica (cosmovisión) referida a la naturaleza del ecosistema y la racionalidad instrumental (metas específicas y herramientas de gestión) que se encentra en la legislación peruana sobre calidad ambiental, es posible que se deba a una escasa reflexión epistemológica en el proceso de formulación de los dispositivos legales, reflexión que debería servir para armonizar la cosmovisión que se tiene con la racionalidad instrumental que se usa para su implementación.

- Una situación alternativa - y no excluyente de la comentada en el párrafo anterior - puede corresponder a que en el presente momento se registra en la legislación ambiental peruana un proceso de competencia entre los paradigmas de la naturaleza estacionaria y de la naturaleza evolutiva. De ser esta la situación, una tarea que se tendrá que acometer es la indagación de cuál de los dos tiene exceso de contenido empírico sobre su competidor, es decir cuál permite ver - entender, tal vez sea mejor - una mayor cantidad de hechos.

\section{Agradecimientos.}

Los autores quieren agradecer al Programa Nacional de Innovación para la Competitividad y Productividad del Ministerio de la Producción por haber financiado el Proyecto "Determinación de Criterios para el Establecimiento de Estándares de Calidad Ambiental para la Diversidad Biológica. Estudio de Caso: Distrito de Cajatambo”. Contrato No. 365-PNCIP-PIAP-2014. Al amparo de dicho proyecto se ha desarrollado la presente investigación.

Al mismo tiempo se desea agradecer a todos los miembros del equipo del mencionado proyecto, en particular a Zulema Quinteros y a Viviana Castro, por el apoyo prestado.

\section{Literatura citada.}

Andréu J. 1998. Los españoles: Opinión sobre sí mismo, España y el Mundo. Análisis Longitudinal Escala de Cantril. Editorial Universidad de Granada.

Beisner B.E., Haydon D.T. \& Cuddington K. 2003. Alternative stable states in ecology. Front Ecol Environ. 1(7): 376-382.

Berelson B. 1952. Content Analysis in Comunication Research, Free Press, Glencoe.

Boyle M., Kay J. \& Pond B. 2001. Monitoring in support of Policy: an Adaptive Ecosystem Approach. 4: 116-137. En T.Munn (ed), Encyclopedia of Global Environmental Change. John Wiley and Son.

Cumming G. S. \& Collier J. 2005. Change and identity in complex systems. Ecology and Society 10(1):29. [online] http://www.ecologyandsociety.org/vol10/iss1/art29/

Holling C.S., Gunderson L. H. \& Ludwig D. 2001. In Quest of a theory of adaptive change. Páginas 3-24. En Panarchy: Understanding transformations in human and natural systems. L. H. Gunderson \& C.S. Holling (editores). Island Press, Washington, DC.

Chapin F. S. III, Kofinas G.P. \& Folke C. (Editores). 2009. Principles of Ecosystem Stewardship: Resilience-Based Natural Resource Management in a Changing World. DOI 10.1007/978-0-387-73033-2. Springer Science+Business Media, LLC.

De Leo G. A. \& Levin S. 1997. The multifaceted aspects of ecosystem integrity. Conservation Ecology 1(1): 3. URL: http://www.consecol.org/vol1/iss1/art3/

Folke C., Carpenter S. R., Walker B., Scheffer M., Chapin T. \& Rockström J. 2010. Resilience thinking: integrating resilience, adaptability and transformability. Ecology and Society 15(4): $20 . \quad$ URL: http:// www.ecologyandsociety.org/vol15/iss4/art20/

Green D., Klomp N., Rimmington G. \& Sadedin S. 2006. The imbalance of Nature. En: Complexity in Landscape Ecology. Chapter 5. Springer Verlag.

Holling C.S. 1998. Two cultures of ecology. Conservation $\begin{array}{llll}\text { Ecology } & \text { 2(2): } & 4 .\end{array}$ http://www.consecol.org/vol2/iss2/art4/

Hull R. B., Robertson D. P., Richert D., Seekamp E. \& Buhyoff G. J. 2002. Assumptions about ecological scale and nature knowing best hiding in environmental decisions. Conservation Ecology 6(2): 12. URL: http://www.consecol.org/vol6/iss2/art12

Kay J., Regier H., Boyle M. \& Francis G. 1999. An ecosystem approach for sustainability: addressing the challenge of complexity. Futures. 31 (7): 721-742.

Kuhn T. S. 2006. La Estructura de las Revoluciones Científicas. C. Solís (Trad.) 3ª. Ed. Fondo de Cultura Económica, México. 
Lakatos I. 1989. La Metodología de los Programas de Investigación Científica. Alianza Editorial, Madrid.

March J. M. 2005. Nuevos Fundamentos de Racionalidad Ambiental a partir del Análisis Epistemológico de la Evaluación de Impacto Ambiental. Cinta de Moebio, número 024. Universidad de Chile, Santiago.

Marshall A. 1998. A Postmodern Natural History of the World: Eviscerating the GUTs from Ecology and Environmentalism. Stud. Hist. Phil. Biol. \& Biomed. Sci. 29: 137-164.

Nuñez M. \& Nuñez P., 2005. Controversias en ecología: la competencia, de la certeza a la pregunta. Ecología Austral 15:229-238. Asociación Argentina de Ecología.

Odenbaugh, J. 2001. Ecological Stability, Model Building, and Environmental Policy: A Reply to Some of the Pessimism. Philosophy of Science.68.

Perú, Ministerio del Ambiente. sf. Compendio de la Legislación Ambiental Peruana. Calidad Ambiental. Volumen V. Dirección General de Políticas, Normas e Instrumentos de Gestión Ambiental. Documento Preliminar.
Ulanowicz R. 2007. Ecology, a Dialog between the Quick and the Dead. 1:27-46 En: F. Capra, A. Juarrero, P. Sotolongo \& J. Vand Uden (editores) Reframing complexity: Perspectives from the North and the South. Exploring Complexity Book. ISCE Publishing, 395 Central Street, Mansfield, MA 02048, USA.

Walker B., Holling C. S., Carpenter S.R. \& Kinzig A. 2004. Resilience, adaptability and transformability in socialecological systems. Ecology and Society 9(2): 5. URL: http://www.ecologyandsociety.org/vol9/iss2/art5

Walker B., Carpenter S. R. , Anderies J., Abel N., Cumming G., Janssen M., Lebel L., Norberg J., Peterson G.D. \& R. Pritchard R. 2002. Resilience management in social-ecological systems: a working hypothesis for a participatory approach. Conservation Ecology 6(1): 14. URL: http://www.consecol.org/vol6/iss1/art14

Weber M. 1951. Soziologische Grundbegriffe. En: Gesammelte Aufsätze zur Wissenschaftslehre. Edit. J. Winckelmann.

Anexo 1. Dispositivos legales de la normativa peruana sobre calidad ambiental, sometidos a análisis.

\begin{tabular}{|c|c|}
\hline DISPOSITIVO LEGAL & OBJETO DEL DISPOSITIVO LEGAL- \\
\hline DECRETO SUPREMO Nº 074-2001-PCM & le Estándares Nacionales de Calidad Ambiental del Aire. \\
\hline DECRETO SUPREMO No 002-2008-MINAM & Aprobación de los Estándares Nacionales de Calidad Ambiental para Agua. \\
\hline DECRETO SUPREMO Nº 010-2005-PCM & Estándares de Calidad Ambiental para Radiaciones No Ionizantes. \\
\hline DECRETO SUPREMO Nº 069-2003-PCM & Establecen Valor Anual de Concentración de Plomo. \\
\hline DECRETO SUPREMO Nº 085-2003-PCM & Reglamento de Estándares Nacionales de Calidad Ambiental para Ruido. \\
\hline CONSTITUCION POLÍTICA DEL PERÚ & $\begin{array}{l}\text { Constitución Política del Perú (incluye sólo los artículos concernientes al medio } \\
\text { ambiente). }\end{array}$ \\
\hline DECRETO LEGISLATIVO Nº 757 & $\begin{array}{l}\text { Ley Marco para el Crecimiento de la Inversión Privada. Título VI. De la Seguridad } \\
\text { Jurídica de la Conservación del Medio Ambiente. }\end{array}$ \\
\hline DECRETO LEGISLATIVO N 1039 & $\begin{array}{l}\text { Modificación de algunas disposiciones del Decreto Legislativo No. } 1013 \text { (Creación } \\
\text { del MINAM) }\end{array}$ \\
\hline DECRETO LEGISLATIVO Nº 1013 & Ley de Creación, Organización y Funciones del Ministerio del Ambiente. \\
\hline DECRETO LEGISLATIVO No 1055 & Modificación de la Ley Nº 28611, Ley General del Ambiente. \\
\hline DECRETO SUPREMO Nº 006-2009-MINAM & Precisan denominación de la Comisión Nacional sobre el Cambio Climático. \\
\hline DECRETO SUPREMO Nº08-2005-PCM & $\begin{array}{l}\text { Reglamento de la Ley } N^{0} \text { 28245, Ley Marco del Sistema Nacional de Gestión } \\
\text { Ambiental. }\end{array}$ \\
\hline DECRETO SUPREMO N ${ }^{\circ}$ 086-2003-PCM. & Estrategia Nacional sobre Cambio Climático. \\
\hline DECRETO SUPREMO Nº 102-2001-PCM & Aprueban Estrategia Nacional de la Diversidad Biológica del Perú. \\
\hline LEY N ${ }^{\circ} 28611$ & Ley General del Ambiente \\
\hline LEY Nº 26821 & Ley Orgánica para el Aprovechamiento Sostenible de los Recursos Naturales. \\
\hline LEY Nº 28245 & Ley Marco del Sistema Nacional de Gestión Ambiental. \\
\hline DECRETO SUPREMO No 003-2008-MINAM & Estándares Nacionales de Calidad para el Aire. \\
\hline DECRETO SUPREMO Nº 023-2009-MINAM. & Implementación de los Estándares de Calidad Ambiental para el Agua. \\
\hline DECRETO SUPREMO Nº 002-2013-MINAM & Aprueban Estándares de Calidad Ambiental para el Suelo. \\
\hline DECRETO SUPREMO No 009-2012-MINAM & $\begin{array}{l}\text { Límites Máximos Permisibles de Emisiones Contaminantes para Vehículos } \\
\text { Automotores que circulen en la Red Vial. }\end{array}$ \\
\hline DECRETO SUPREMO N ${ }^{\circ}$ 003-2010-MINAM & $\begin{array}{l}\text { Límites Máximos Permisibles para los efluentes de Plantas de Tratamiento de } \\
\text { Aguas Residuales Domésticas o Municipales. }\end{array}$ \\
\hline DECRETO SUPREMO No 010-2010-MINAM & $\begin{array}{l}\text { Límites Máximos Permisibles para la descarga de efluentes líquidos de Actividades } \\
\text { Minero - Metalúrgicas. }\end{array}$ \\
\hline
\end{tabular}


Anexo 2. Conceptos que se han usado en el análisis. Se los ha separado en tres niveles (Objetivos generales de la gestión, Naturaleza del ecosistema y Herramientas de Gestión). Para los dos últimos niveles se han separado lo conceptos en función del paradigma al que pertenecen.

\begin{tabular}{|c|c|c|}
\hline Objetivos general de la Gestión. & & \\
\hline Diversidad & & \\
\hline Gestión & & \\
\hline Desarrollo Sostenible & & \\
\hline Calidad ambiental & & \\
\hline Cambio climático & & \\
\hline Procedimiento Administrativo & & \\
\hline Contaminación & & \\
\hline Manejo & & \\
\hline Salud humana & & \\
\hline Salud del ecosistema & & \\
\hline Valores de referencia & & \\
\hline Rendimientos & & \\
\hline Naturaleza del ecosistema & & \\
\hline Atributos & Naturaleza Estacionaria & Gestión de la Resiliencia \\
\hline Tipo de estructura & Máquina & Ecosistema \\
\hline & Mecanismo & Sistema \\
\hline Tipo de causalidad & Procesos unicausales & Procesos multi-causa \\
\hline Variables importantes & Organización por Factores bióticos. & Organización por factores biofísicos \\
\hline Nivel de complejidad & Simplicidad & Complejidad \\
\hline & & Relaciones Multi-escala \\
\hline Procesos que operan & Organización & Auto-organización \\
\hline & & Emergencia (de propiedades) \\
\hline & & Retroalimentación \\
\hline Comportamiento en el tiempo & Estabilidad & Cambio \\
\hline & & Evolución \\
\hline & & Múltiples atractores \\
\hline & & Aprendizaje \\
\hline Predecibilidad & Predecibilidad (predicción) & Impredecibilidad \\
\hline Diferenciación & Homogeneidad & Heterogeneidad \\
\hline & Uniformidad & \\
\hline $\begin{array}{l}\text { Herramientas de Gestión del } \\
\text { ecosistema }\end{array}$ & & \\
\hline & & Gestión de la \\
\hline Atributos & Naturaleza Estacionaria & Resiliencia \\
\hline Tipo de gestión & Manejo de un solo recurso & $\begin{array}{l}\text { Manejo de múltiples recursos } \\
\text { Administración del ecosistema }\end{array}$ \\
\hline Objetivo & Rentabilidad económica & Integridad del ecosistema \\
\hline Metas a conseguir & Máximo (rendimiento) & Óptimo (Rendimiento) \\
\hline & Mínimos & \\
\hline Métodos & Análisis & Síntesis \\
\hline Instrumentos & Estándares de Calidad Ambiental & Monitoreo \\
\hline & Límites Máximos Permisibles & Adaptación \\
\hline & & Flexibilidad \\
\hline & & Contexto-dependencia \\
\hline Rol de las perturbaciones & Impactos (Control) & Impactos (Promoción) \\
\hline & Perturbaciones (Control) & Perturbaciones (Promoción) \\
\hline Tipo de participación & Proceso participativo (gobierno) & Participación ciudadana \\
\hline & & Consenso \\
\hline
\end{tabular}

\footnotetext{
${ }^{1}$ Laboratorio de Ecología de Procesos LEP. Universidad Nacional Agraria la Molina, Av. La Molina S/N. La Molina - Lima Perú. esi@lamolina.edu.pe

2 Investigadora asociada al Laboratorio de Ecología de Procesos LEP. Universidad Nacional Agraria la Molina, Av. La Molina S/N. La Molina - Lima Perú. marizaperu16@yahoo.es
} 